\title{
Linking monoterpenes and abiotic stress resistance in grapevines
}

\author{
Massimo Bertamini ${ }^{*}, 1,2$, Maria Stella Grando ${ }^{1,2}$, Pietro Zocca ${ }^{2}$, Michele Pedrotti ${ }^{2,3}$, Silvia Lorenzi ${ }^{2}$, and Luca \\ Cappellin $^{2}$ \\ ${ }^{1}$ Center Agriculture Food Environment, University of Trento, Via E. Mach, 1, 38010 San Michele a/A, Italy \\ ${ }^{2}$ Research and Innovation Centre, Fondazione Edmund Mach, Via E. Mach, 1, 38010 San Michele a/A, Italy \\ ${ }^{3}$ Wageningen University, Department of Food Quality and Design, P.O. Box 8129, 6700 EV Wageningen, The Netherlands
}

\begin{abstract}
Rising temperatures and ozone levels are among the most striking stressful phenomena of global climate changes, and they threaten plants that are unable to react rapidly and efficiently. Generic responses of plants to stresses include the production of excess reactive oxygen species (ROS). Excessive ROS accumulation can lead to extensive oxidation of important components such as nucleic acids, proteins and lipids which can further exacerbate ROS accumulation leading to programmed cell death. Although most studies on plant antioxidants have focused on non-volatile compounds, volatiles belonging to the isoprenoid family have been implicated in the protection against abiotic stresses, in particular thermal and oxidative stress whose frequency and extent is being exacerbated by ongoing global change and anthropogenic pollution. Historically, research has focused on isoprene, demonstrating that isoprene-emitting plants are more tolerant to ozone exposure and heat stress, reducing ROS accumulation. Yet, evidence is being compiled that shows other volatile isoprenoids may be involved in plant responses against abiotic stresses. Grapevines are not isoprene emitters but some varieties produce other volatile isoprenoids such as monoterpenes. We investigated photosynthesis and emission of volatile organic compounds upon heat stress in two Vitis vinifera cv. 'Chardonnay' clones differing only for a mutation in the DXS gene (2-C-methyl-D-erythritol 4-phosphate (MEP) pathway), regulating volatile isoprenoid biosynthesis. We showed that the mutation led to a strong increase in monoterpene emission upon heat stress. At the same time, maximum photochemical quantum yield ( $\mathrm{Fv} / \mathrm{Fm}$ ratio) of PSII was affected by the stress in the non-emitting clone while the monoterpene emitter showed a significant resilience, thus indicating a possible antioxidant role of monoterpenes in grapevine. Future mechanistic studies should focus on unveiling the actual mechanism responsible for such findings.
\end{abstract}

Corresponding author: massimo.bertamini@unitn.it 


\section{Introduction}

Climate changes, and in particular global warming, affect many agricultural fields. According to the Intergovernmental Panel on Climate Change's (IPCC) Fifth Assessment Report, climate change over the past decades is unequivocal and will continue in the following century [1]. Grape growth and wine production are highly dependent on climatic conditions. The effect of climate may depend on different factors, and has been studied through modeling [2] and field experimentation. Specifically, the impact of high temperatures was modeled by [3]. In viticulture, global warming has proved to be an important obstacle to maintaining production levels and especially the quality of the grapes and wines in the Mediterranean regions [4]. Climate changes strongly impact the physiology of the grapevine: phenology is mainly influenced by temperature and this aspect can strongly impact varietal adaptation to different environments [5].

The increase in canopy temperature is the main cause of abiotic stresses such as heat stress and water stress. Thermal stress becomes a challenge in viticulture when the heat dissipation from the foliage is less than the absorbed solar energy, particularly if the water supply is not sufficient to disperse latent heat with the leaf transpiration, thus leading to irreversible changes in the plants [6]. The risk of heat stress occurrence depends on the likelihood of high temperatures and their duration during the day or night. Plants can be damaged in different ways both during day or nighttime hours, in the presence of high ground temperature or hot air. High temperatures can have direct harmful effects associated with physical damage to tissues or indirect effects associated with changes in metabolism [7]. One of the main consequences of heat stress due to the presence of strong solar radiation is the excessive generation of reactive oxygen species (ROS), which leads to oxidative stress [8].

The plants are able, in different measures, to tolerate heat stress both through physical and morphological changes of the plant itself, and often, generating signals for the metabolism to change. Metabolic changes can occur in different ways in response to heat stress: producing compatible solutes that can organize proteins and cellular structures, maintaining cellular turgidity through osmotic adjustment, modifying the antioxidant system to restore the cellular redox balance and homeostasis [9]. In general, heat stress is responsible for the up-regulation of several heat-inducing genes, commonly called "heat shock genes" (HSG) that encode HSP proteins, and these active products are very necessary for plant survival under conditions of fatal heat [6].

High temperature also has a strong influence on the photosynthetic capacity of plants, especially in $\mathrm{C} 3$ plants compared to $\mathrm{C} 4$ plants. In chloroplast, carbon metabolism of the stroma and photochemical reactions in thylakoid membranes are considered as the primary sites of injury at high temperatures $[10 ; 11 ; 12]$. The thylakoid membranes are highly susceptible to heat stress: thermal shock reduces or even interrupts the activity of photosystem II (PSII). Specifically the photochemical quantum yield of PSII, measured as $\mathrm{Fv} / \mathrm{FM}$ ratio, is reduced by the same stress condition (13; 14].

Many plant species respond to abiotic stress through the formation of volatile organic compounds (VOCs). Abiotic stresses can induce changes in constitutive VOCs either stimulating or suppressing emissions [15; 16]. Although numerous studies have indicated that VOCs may have an important regulatory role within a plant, the biological functions of some of these volatile compounds are still poorly understood. [17]. Terpenes are the main VOCs produced by plants under thermal stress, at temperatures ranging from 20 to $40^{\circ} \mathrm{C}$ [18; 19]. It is well known that terpenes are emitted from plant leaves under wounding, insect or herbivore attacks [20]. The effect of biotic interactions on VOC emissions from plants and long-distance signaling in plant defense has been studied intensively during the last two decades [21; $22 ; 23 ; 24]$. A role of isoprene and monoterpenes has been highlighted in the protection of plants against abiotic stresses [25]. Terpenes may protect the plant from heat damage but if the heat stress occurs with temperature above the optimal enzyme temperature (around $40-45^{\circ} \mathrm{C}$ for enzymes in the MEP pathway and most TPS), there might be a very rapid inhibition of terpene synthesis [16] evidenced that oxidative and thermal stresses are relieved in the presence of biogenic volatile organic compounds like terpenes. Many studies have been focusing on isoprene as this is the most emitted hydrocarbon by plants into the atmosphere [26]. Grapevines have not been reported to emit isoprene, but some varieties do emit monoterpenes [27]. The emission capacity of VOCs, especially terpenes, from vine leaves of aromatic varieties has not been sufficiently explored. This is important because some VOCs might protect the plant from abiotic stress, acting as an antioxidant, as it has previously been proposed [28].

\section{Aim of the study}

The aim of the present work was to evaluate the effects of acute heat stress on the emission of volatile monoterpenes from the leaves of Chardonnay grapes and a possible correlation with stress resistance. Two Chardonnay clones were compared: the ENTAVINRA ${ }^{\circledR} 809$ musque clone and the SMA ${ }^{\circledR} 130$ nonaromatic clone [29]. These two varieties were chosen because genetically they only differ by a mutation in the monoterpene pathway and therefore represent a meaningful study case. In particular, we wanted to evaluate: if the two clones, which differ in terpenoid accumulations of the grapes, are also characterized by different terpene emission as a VOCs from leaves; if this emission of VOCs can be related to the condition of acute high temperature stress (HTS); and, most importantly, if such VOCs are correlated with the activation of defense mechanisms capable of reducing the limitation of the photochemical quantum yield of PSII in HTS conditions.

Corresponding author: massimo.bertamini@unitn.it 


\section{Materials and methods}

\subsection{Plant materials}

The trial was conducted in the years 2017 and 2018. Two Chardonnay aromatic and non-aromatic clones were compared [29]: The ENTAV-INRA ${ }^{\circledR} 809$ Chardonnay clone was selected in 2004 and is considered an aromatic clone of type 'musqué,, i.e. having grapes and musts reminiscent of Muscat variety; The SMA ${ }^{\circledR} 130$ Chardonnay clone was selected at the Agricultural Experimental Station of San Michele all'Adige (Trento), in 1978. It is characterized by good productivity, good level of organic acids and sugars, and absence of terpenic compounds in grapes and must.

The musque clone differs from the neutral clone by a single nucleotide (SNP) in the VvDXS gene that Emanuelli et al (2010) found is associated with the aroma of the grapes. The SNP $(1784 \mathrm{~T}>\mathrm{C})$ involves the replacement of an amino acid in the relative enzyme and is now easily diagnosed with various molecular techniques. The Chardonnay clones are cultivated in the polyclonal vineyard (Weizsäcker) located in the agricultural holding of the Mach Foundation at San Michele all'Adige (46th 11'37 "68 N, 11 ${ }^{\circ} 8^{\prime} 4 " 56$ E, at $232 \mathrm{~m}$ of altitude).

The vineyard was planted in 2004, with a $15-20 \%$ slope, west exposure, calcareous skeletal soils, with a loam-limestone texture, $15 \%$ clay, low endowment of organic substance and balanced content of nutritive elements. Density of planting was 5.600 plants / hectare and vines were pruned to a Guyot system. The clonal identity of individual plants was verified at the level of the SNP1784 to guarantee the reliability of the comparison.

\subsection{VOC measurement}

The experiments were carried out on grapevine shoots collected from the field the same day of the measurement. The plants were immediately placed in water within a climatic chamber (Climacell 707, BMT Medical Technology s.r.o, Brno, Czech Republic). Each experimental day, only plants belonging to the same cultivar were measured in order to avoid plant-plant communication during the experiments. Measurements were performed in five replicates per year. Each plant was enclosed using a Teflon PFA bag. About seven fully developed leaves were enclosed. A commercial Gas Calibration Unit instrument (Ionicon Analytik $\mathrm{GmbH}$ ) was employed to generate zero air which was delivered to each bag at a constant flow of about $350 \mathrm{sccm}$. A PEEK capillary delivered the enclosed air to a commercial PTR-TOF-MS 8000 instrument (Ionicon Analytik GmbH, Innsbruck, Austria). An overflow allowed excess air to exit the enclosure. The temperature treatment described in the following section was performed after two hours, during which monoterpene emission stabilized at the constitutive level. During the measurements, an automated inlet switching system allowed the PTR-ToF-MS to cycle between each enclosure every 12 minutes.

\subsection{High Temperature Stress (HTS) treatments}

The HTS treatment consisted of the following temperature steps of climatic chamber: starting temperature of $25^{\circ} \mathrm{C}$ for 1 hour and 30 minutes, followed by an increase to $52^{\circ} \mathrm{C}$ for 2 hours and reducing temperature down to $25^{\circ} \mathrm{C}$ for 2 hours. $\mathrm{RH}$ and light intensity were kept constant at $70 \%$ and $270 \mu \mathrm{mol} \mathrm{m} \mathrm{m}^{-2}$, respectively.

\subsection{Data analysis}

To extract VOC concentrations from the PTR-ToF-MS raw data, the softwares TOFOcal, TOFOpeak (developed in-house at the Fondazione Edmund Mach), as well as other scripts developed in MATLAB (MathWorks, Natick, MA) were used. Non-parametric test MannWhitney with p-value $<0.01$ was employed to determine significant differences between the two varieties.

\subsection{Quantum yield of PSII efficiency (Fv/FM) measurements}

The chlorophyll fluorescence was measured on the upper leaf surface using PAM 2000 fluorometer (Heinz Walz, Effeltrich, FRG). Before the measurement, the leaves were adapted to dark for 30'. Fo were measured by switching on the modulated light $0.6 \mathrm{Khz}$; Photosynthetic Photon Flux Density (PPFD) was less than $0.1 \mu \mathrm{E} \mathrm{m} \mathrm{m}^{-2} \mathrm{~s}^{-1}$ at the leaf surface. Fm were measured at $20 \mathrm{kHz}$ with a one saturation pulse of 6000 $\mu \mathrm{mol}$ (photon) $\mathrm{m}^{-2} \mathrm{~s}^{-1}$ of white light [30]. The quantum yield of PSII efficiency was determined as the ratio Fm$\mathrm{Fo} / \mathrm{Fm}(\mathrm{Fv} / \mathrm{Fm})$, Measurements were performed before and after stress. Control plants were employed, six leaves per plant were used for the measurement. Each leaf was measured three times $(3 \times)$. T-test with $p$-value $<0.05$ was performed to determine significant differences between the two varieties.

\section{Results}

\subsection{Year 2017 trial}

In September 2017, HTS treatments were performed on whole shoots taken from the field of the two Chardonnay clones. The total monoterpene emission by the leaves of the two different clones was very low before the HTS (Figure 1). During two hours of HTS treatment we observed a significant increase in VOCs (monoterpens) only by the clone INRA809 while the clone SMA130 continues to present a substantially absent emission.

After two hours of subsequent recovery at unstressed conditions the monoterpene emission in INRA809 was much higher that that of SMA130 (Figure 1).

Before the HTS treatment the photochemical quantum yield of the leaves of the two clones was very similar, with a slight prevalence of the value of the clone 809 (0.756 for SMA130 and 0.769 for INRA 809, Figure 2). After two hours HTS the leaves of the SMA 130 clone showed significantly lower Fv/FM (0.397) values than those of the INRA 809 clone (0.629, Figure 2$)$. 

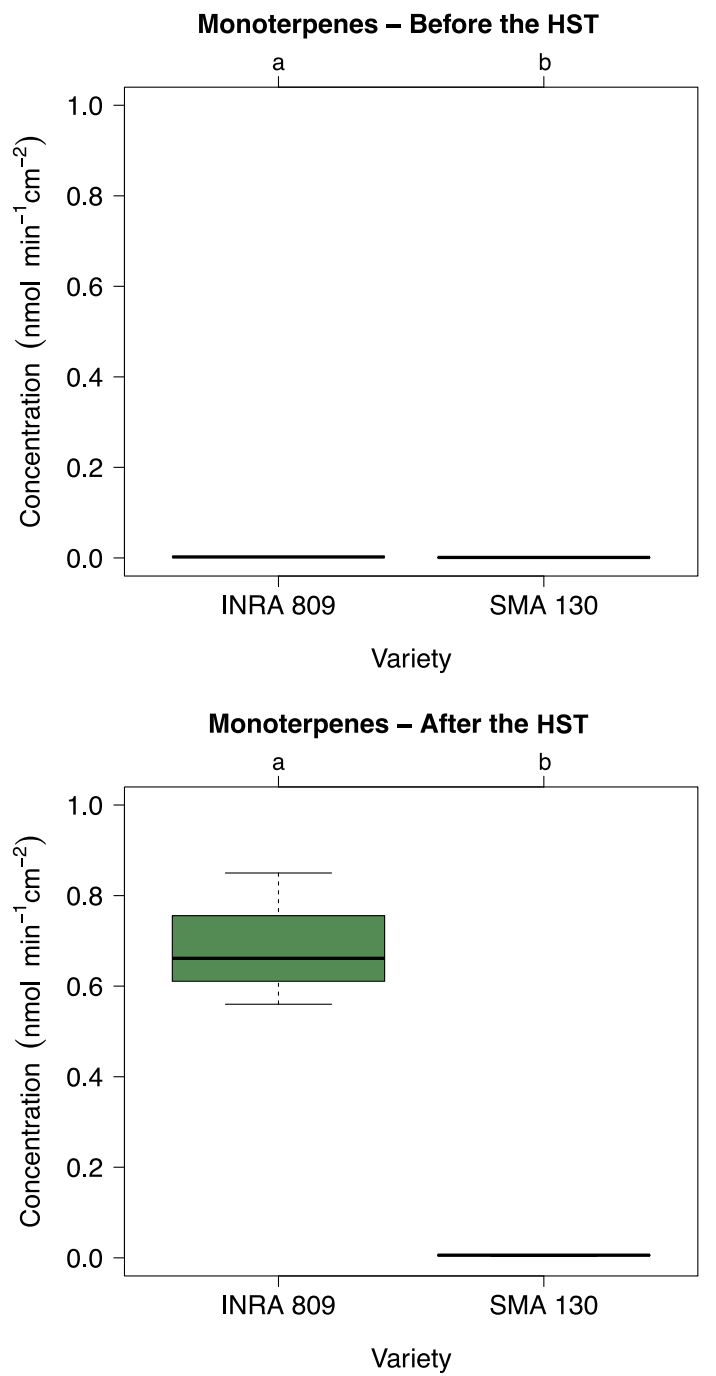

Fig. 1. Monoterpenes emission by SMA 130 and INRA 809 before and after HTS. All measurements were taken at $25^{\circ} \mathrm{C}$. Data from 2017. The emission of the two varieties, when significantly different $(\mathrm{p}<0.01)$, are marked on the top with different letters $(a, b)$.

\subsection{Year 2018 trial}

The experimentation was repeated in June 2018. Ten whole shoots were taken from the vineyard for each clone and subjected to HTS for two hours, measuring the emission of monoterpenes and the photochemical quantum yield of PSII in unstressed conditions and upon HTS. Before HTS, the emission of total monoterpenes was almost absent in both clones (Figure 3). After the two hours of HTS the emission was significantly accentuated, with a more consistent effect on clone INRA 809 which had significantly higher values of monoterpene emission $(\mathrm{p}<0.01)$.

Upon recovering, clone INRA 809 showed a strong increase in monoterpene emissions, but also clone SMA 130 had an increase in emission, although to a significantly lower degree (Figure 3 ).

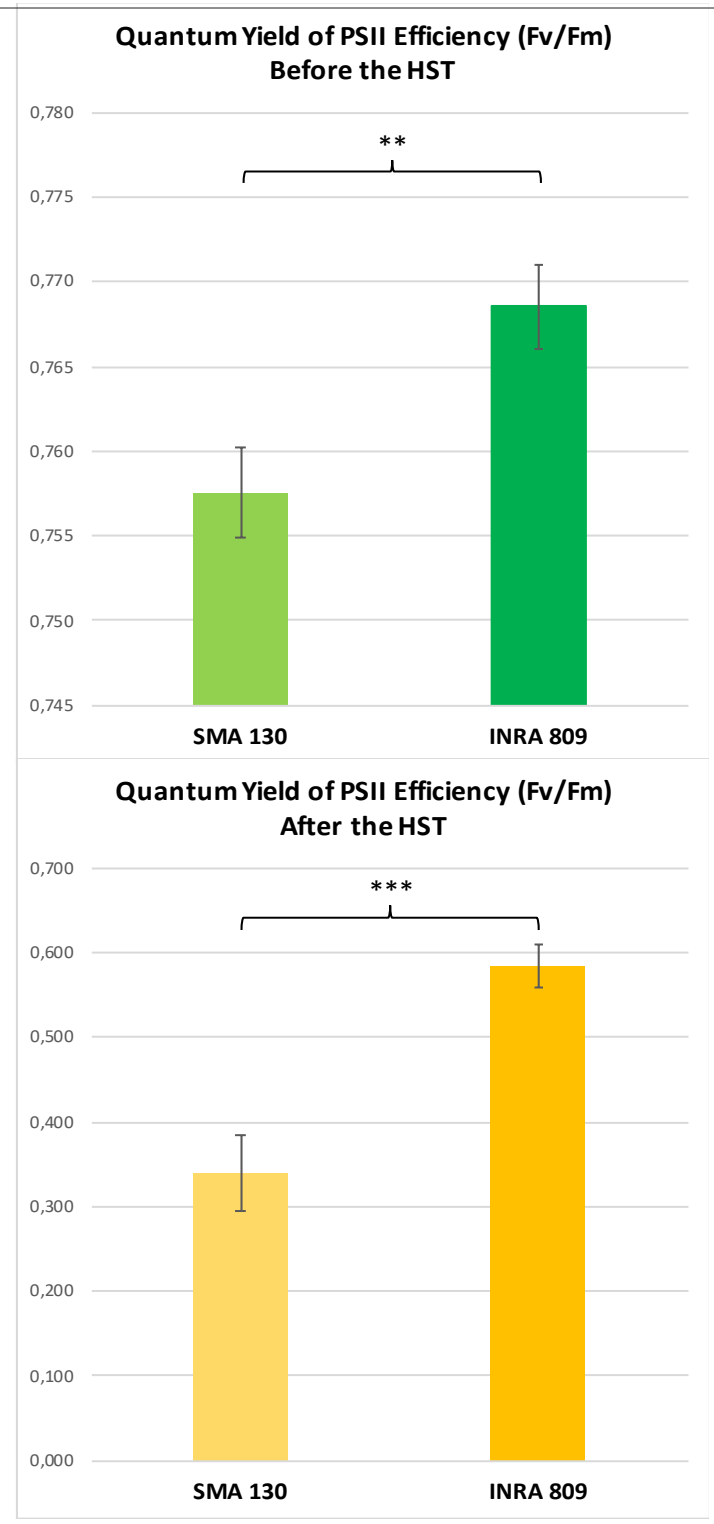

Fig. 2. Monoterpenes emission by SMA 130 and INRA 809 before and after HTS. All measurements were taken at $25^{\circ} \mathrm{C}$. Data from 2017. The emission of the two varieties, when significantly different $(p<0.01)$, are marked on the top with different letters $(a, b)$.

Regarding the photochemical quantum yield of PSII, no significant differences in $\mathrm{Fv} / \mathrm{Fm}$ were found between the two clones before HTS $(0.770$ clone 130 and 0.775 clone 809 , Figure 4$)$. Such results were very similar to the values recorded in the previous year and show that the plants in the fields had good photosynthetic performances, given the high Fv/Fm values. After two hours of HTS the clone 809 still appears as the best performing, with a lower drop of Fv/Fm. The differences appear to be significant (Figure 4).

It is worth noting that the environmental conditions in the two years were very different. In spring 2018, the first months of the vegetative season, the vines were not presented any stressful environmental conditions, as a result of numerous rainy days, absence of water stress and a general lack of particularly high temperatures and intense global radiation. To the contrary, the 2018 growing season was very dry with prolonged periods of 
high temperatures. Nonetheless, the results of the two years are consistent.
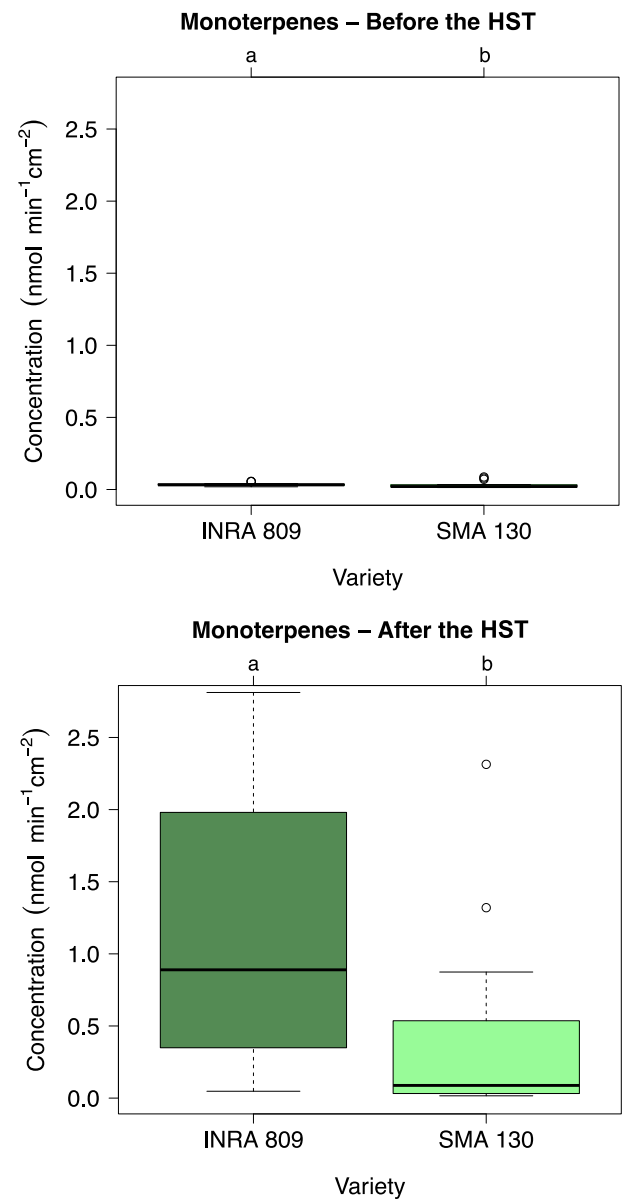

Fig. 3. Monoterpene emission from SMA 130 and INRA809 before and after HTS. All measurements were taken at $25^{\circ} \mathrm{C}$. Data from 2018. The emission of the two varieties, when significantly different $(\mathrm{p}<0.01)$, are marked on the top with different letters $(a, b)$.

It is interesting to separate the results between adult leaves and young leaves as presented below (Figure 5 and 6). It was shown that the significant difference found between the clones is limited to the adult leaves $(0.110$ for INRA 809 compared to 0.056 for SMA 130, Figure 5).To the contrary, the leaves of the clone 809 during ontogenetic development (less than 30 days old) seem to indicate a similar damage of the PSII under HST conditions to the leaves of the clone 130 (Figure 6).

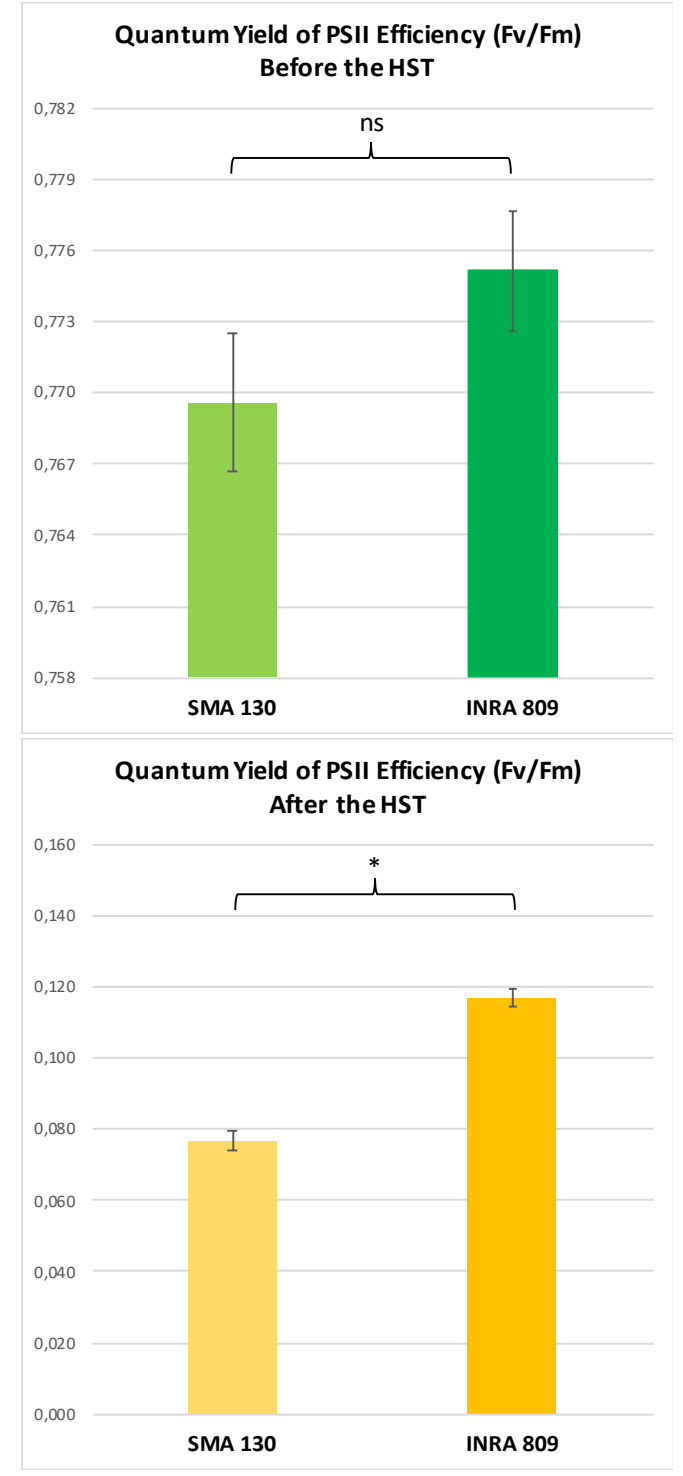

Fig. 4. Photochemical quantum yield of SMA 130 and INRA 809 before and after HTS. All measurements were taken after 30 ' in the dark. Data from 2018. '*': significant difference $(p<0.05)$; 'ns': non-significant difference.

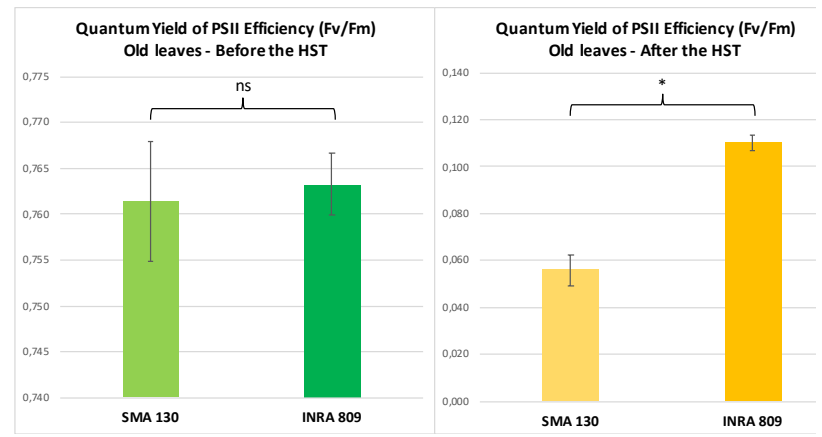

Fig. 5. Photochemical quantum yield of SMA 130 and INRA 809 older leaves, before and after HTS. All measurements were taken after 30' in the dark. Data from 2018 


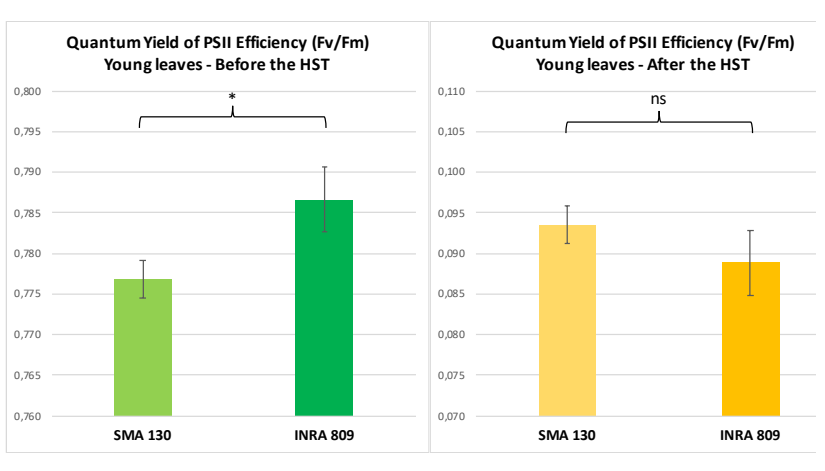

Fig. 6. Photochemical quantum yield of SMA 130 and INRA 809 younger leaves, before and after HTS. All measurements were taken after $30^{\prime}$ in the dark. Data from 2018.

\section{Conclusions and future perspectives}

These preliminary experimental findings have provided useful information to the questions posed in the objectives of this work while creating further questions that must still be investigated. The work showed that the two Chardonnay clones, which show genetic differences in terms of terpene synthesis capacity in berries, are also characterized by different terpene emissions from the leaves. Specifically, the INRA 809 clone emits a significantly higher number of monoterpenes if the plants are subjected to acute thermal shock for two hours compared to the SMA130 clone. The monoterpene emission appears to be related to the acute thermal stress condition (HTS) and correlated with the activation of defense mechanisms able to limit the reduction of the photochemical quantum yield of PSII (measured as Fv/FM values). Such results are remarkable and consistent with findings in other plants and for other terpenoids such as isoprene (Loreto and Schnitzler, 2010). It is the very first time that a connection between monoterpene emission and heat stress resistance is reported in grapevines. The two Chardonnay clones represented a very meaningful case study as the only genetic difference between them was a mutation in a gene belonging the MEP pathway, responsible for monoterpene biosynthesis.

The present investigation does not shed light on the actual mechanisms responsible for such effect. To our knowledge, the production of monoterpenes by the leaves subjected to HTS has not been reported to have a direct influence on the photoinhibition reduction mechanisms. Rather, it is mediated by the increased ability to counteract the negative effect of ROS either directly via scavenging, or indirectly via thylakoid membrane stabilization. It is also possible that monoterpenes act as a signaling molecule. In analogy with recent considerations on the antioxidant role of isoprene [31], the latter hypothesis seems the most likely. However, further investigation should be performed in order to clarify this aspect.

It also remains to be clarified how the foliar ontogenetic development phases influence the response both in terms of VOCs emission and of photochemical quantum yield of PSII activity. In young leaves during HTS conditions it is likely that a higher quantum yield of PSII is not necessarily positive. In fact, a quenching of the efficiency of the PSII reaction center could be positive to reduce ROS formation if the plant has not activated the necessary defenses against such compounds yet.

Luca Cappellin acknowledges funding from H2020-EU.1.3.2 (grant agreement n. 659315).

\section{Bibliography}

1. Intergovernmental Panel on Climate Change (IPCC). Summary for policymakers. In Stocker, T.F., Qin, D., Plattner, G.-K., Tignor, M., Allen, S.K., Boschung, J., Nauels, A., Xia, Y., Bex, V., and Midgley, P.M. (Eds.), Climate Change 2013: The Physical Science Basis. Contribution of Working Group I to the Fifth Assessment Report of the Intergovernmental Panel on Climate Change. Cambridge (Cambridge University Press, 3-29, 2013)

2. P. Piéri and E. Lebon, Journal International des Sciences de la Vigne et du Vin, 35-43 (2014).

3. P. Piéri, Changement climatique et culture de la vigne: l'essentiel des impacts. In Brisson, N., and F. Levrault (Eds.), Livre Vert du projet CLIMATOR (2007-2010): Changement Climatique, Agriculture et Forêt en France: Simulations d'Impacts sur les Principales Espèces. (Angers, France: Ademe Editions, 213-223, 2010).

4. L. Hannah, P.R. Roehrdanz, M. Ikegami, A.V. Shepard, M.R. Shaw, G. Tabor, L. Zhi, P.A. Marquet, R.J. Hijmans, Proceedings of the National Academy of Sciences, 110(17), 69076912 (2013).

5. I. García de Cortázar-Atauri, É. Duchêne, A. Destrac- Irvine, G. Barbeau, L. de Rességuier, T. Lacombe, A.K. Parker, N. Saurin, C. van Leeuwen, OENO One, 51(2), (2017) DOI : 10.20870/oeno-one.2016.0.0.1622.

6. L.C. Carvalho, J.L. Coito, S. Colaço, M. Sangiogo and S. Amâncio, Plant, Cell \& Environment, 38(4), 777-789 (2015)

7. C.E. Bita, T. Gerats, Front Plant Sci., 31; 4-273 (2013)

8. M. Hasanuzzaman, K. Nahar, M.M. Alam, R. Roychowdhury and M. Fujita, Int. J. Mol. Sci., 14(5) 9643-9684 (2013)

9. A.L. Qu, Y.F. Ding, Q. Jiang, C. Zhu, Biochem. Bioph. Res. Co., 432(2), .203-207 (2013)

10. J.Z. Wang, L.J. Cui, Y. Wang, J.L. Li, Biol. Plant., 53, 247-242 (2009)

11. M. Bertamini and N. Nedunchezhian, J. Plant Physiol., 159, 799-803 (2002)

12. D.H. Greer, M.M. Weedon, Plant Cell. Environ., 35, 1050-1064 (2012). 
13. D. Morales, P. Rodriguez, J. Dellamico, E. Nicolas, A. Torrecillas, M.J. SanchezBlanco, Biol. Plant. 47,203-208 (2003).

14. A. Ferrandino, C. Lovisolo, Environ. Exp. Bot., 103, 138-147 (2014)

15. Blande J. D, Holopainen J.K. \& Niinemets Ü. (2014). Plant volatiles in polluted atmospheres: stress responses and signal degradation. Plant, Cell and Environment (2014) 37, 1892-1904.

16. F. Loreto, J.P. Schnitzler, Trends Plant Sci., 15 (3), 154-166 (2010)

17. J.K. Holopainen, Trends in Plant Sci., 9, 529533 (2004)

18. R.K. Monson, C. H. Jaeger, W.W. Adams, E.M. Driggers, G.M. Silver and R. Fall, Plant Physiol, 98(3), 1175-1180 (1992)

19. M.A. Ibrahim, M. Mäenpää, V. Hassinen, S. Kontunen-Soppela, L. Malec, M. Rousi, L. Pietikäinen, A. Tervahauta, S. Kärenlampi, J.K. Holopainen, E.J. Oksanen, J Exp Bot., 61(6), 1583-95 (2010)

20. B. Wielkopolan and A. Obrępalska-Stęplowska, Planta, 244(2):313-32. doi: 10.1007/s00425016-2543-1. Epub 2016 May 11. Review. (2016)

21. S.P. Gouinguene and T.C.J. Turlings, , Plant Physiol., 129, 1296-1307 (2002)

22. A. Vallat, H.N. Gu, S. Dorn, Phytochemistry, 66, 1540-1550 (2005)

23. J. Gershenzon, Proceedings of the National Academy of Sciences, USA, 104, 5257-5258 (2007)

24. M. Heil, J. Ton, Plant Sci., 2008; 13, 264-272 (2008)

25. V.B. Velikova, J. Plant Interact., 1(3), 1-15 (2008)

26. T.D. Sharkey, A. E. Wiberley and R.D. Autumn, Ann Bot. 101(1), 5-18 (2008)

27. P. Ribéreau-Gayon, J.N. Boidron and A. Terrier, J. Agr. Food Chem., 23(6), 1042-1047 (1975)

28. A. Aharoni, A.P. Giri, S. Deuerlein, F. Griepink, W. J. de Kogel, F.W. Verstappen, H.J. Bouwmeester, The Plant Cell, 15(12), 2866-2884 (2003)

29. E. Duchêne, J.L. Legras, F. Karst, D. Merdinoglu, P. Claudel, N. Jaegli, F. Pelsy, Aust. J. Grape. Wine R., 15(2), 120-130 (2009)

30. M. Bertamini, N. Nedunchezhian, Plant Sci, 164, 635-644 (2003)

31. T.D. Sharkey, R.K. Monson, Plant, Cell \& Environment, 40 (9), 1671-1678 (2017) 